\title{
Evaluation of the Value of Multiplex MicroRNA Analysis as a Breast Cancer Screening in Korean Women under 50 Years of Age with a High Proportion of Dense Breasts
}

\author{
Ji Young Jang ${ }^{1, *}$, Eun Young Ko',*, Ji Soo Jung', Kyung Nam Kang', Yeon Soo Kim², Chul Woo Kim \\ ${ }^{1}$ BIOINFRA Life Science Inc., Seoul, ${ }^{2}$ DIOGENE Inc., Seongnam, Korea
}

\begin{abstract}
This study was conducted to confirm the performance of the microRNA (miRNA) biomarker combination as a new breast cancer screening method in Korean women under the age of 50 with a high percentage of dense breasts. To determine the classification performance of a set of miRNA biomarkers (miR-1246, 202, 21, and 219B) useful for breast cancer screening, we determined whether there was a significant difference between the breast cancer and healthy control groups through box plots and the MannWhitney U-test, which was further examined in detail by age group. To verify the classification performance of the 4 miRNA biomarker set, 4 classification methods (logistic regression, random forest, XGBoost, and generalized linear model plus random forest) were applied, and 10-fold cross-validation was used as a validation method to improve performance stability. We confirmed that the best breast cancer detection performance was achievable in patients under 50 years of age when the set of 4 miRNAs were used. Under the age of 50, the 4 miRNA biomarkers showed the highest performance with a sensitivity of $85.29 \%$, specificity of $93.33 \%$, and area under the curve (AUC) of 0.961. Examining the results of 4 miRNA biomarkers was found to be an effective strategy for diagnosing breast cancer in Korean women under 50 years of age with dense breasts, and hence has the potential as a new breast cancer screening tool. Further validation in an appropriate screening population with large-scale clinical trials is required.
\end{abstract}

Key Words MicroRNA, Breast cancer, Screening, Dense breast, Korean women under the age of 50

\section{INTRODUCTION}

In Korea, breast cancer is the most common malignancy in women. In 2017, there were 22,300 new cases and the crude incidence rate was 86.9 per 100,000 according to data from the Korea National Cancer Incidence Database (KNCID) [1]. The number of incident breast cancers in 2019 was estimated to be at 24,010 , with a crude incidence rate of 92.9 per 100,000 [2], suggesting an increasing trend. An epidemiologic study has suggested that the incidence rate is expected to rapidly increase within the next 10 years in Korea due to the increasing proportion of elderly individuals in the population and the continuous adoption of the Westernized lifestyle [3]. This trend highlights the importance of effective breast cancer screening in Korea.

As an early screening test for asymptomatic people, if there are no specific risk factors, mammography is the best method available. Mammography is the only screening method that has been shown to reduce breast cancer mortality, and the most important benefit of screening mammography is the early detection of breast cancer and reduced mortality. The National Cancer Screening Program (NCSP) in Korea introduced breast cancer screening in 2002 [4]. As of 2015, the NCSP guidelines recommend routine biennial breast cancer screening by mammography for women aged 40 to 69 years, and for women above 70 years old, according to individual preference and risk [5].

Mammography is the primary imaging modality for breast cancer screening worldwide. Despite being acknowledged as a first-line tool, multiple studies have reported that its sensitivity may be as low as $30 \%$ to $48 \%$ for dense breasts [6-8]. The reason is that dense breast tissue can obscure breast cancers on mammography and yield false-negative results. Dense breasts are a well-known risk factor for breast cancer,

Received November 8, 2021, Revised December 9, 2021, Accepted December 22, 2021

Correspondence to Chul Woo Kim, E-mail: chulwoo.kim@bioinfra.co.kr, https://orcid.org/0000-0002-1229-198X

Check for updates

*These authors contributed equally to this work as co-first authors. 
and can also negatively influence the accuracy of breast cancer screening by mammography $[6,9]$. Breast density is defined as the proportion of fibroglandular tissue in the total breast volume. It can be influenced by age, menopausal status, and ethnicity. The proportion of dense breasts tends to be decreased as the patient ages and goes through menopause [9-11].

In Korean women, the frequency of dense breasts according to age was $88.1 \%$ at 30 to 34 years old, $91.1 \%$ at 35 to 39 years old, $78.3 \%$ at 40 to 44 years old, $61.1 \%$ at 45 to 49 years old, $30.1 \%$ at 50 to 54 years old, $21.1 \%$ at 55 to 59 years old, and $7.0 \%$ at 60 to 64 years old. Korean women aged 30 to 49 years showed a very high frequency of high-density breasts, whereas those aged 50 to 54 years had a sharp decrease to $30.1 \%$. Compared to Western women who have high density breasts, $47.2 \%$ for those aged 40 to 44 and $44.8 \%$ for those aged 45 to 49 , Korean women in their 40 s have a very high frequency of high-density breasts $[12,13]$. Mammographic breast density may be the most undervalued and underused risk factor in studies investigating breast cancer occurrence. The risk for breast cancer is four to six times higher in women with dense breasts. Breast density may also decrease the sensitivity and, thus, the accuracy of mammography [14-16].

Accordingly, the limitations of screening mammography for women with dense breasts under the age of 50 are clear. Developing a new high-accuracy breast cancer screening tool in Korean women under the age of 50 with a high proportion of high-density breasts could increase the rate of early breast cancer detection, thereby increasing the therapeutic effect and survival rate. The purpose of this study was to confirm the value of a novel blood-based multiplex miRNA assay as a new adjunct tool for breast cancer screening in Korean women under 50 years of age with high breast density.

\section{MATERIALS AND METHODS}

\section{Cohorts and plasma samples}

In this study, we generated a model to identify breast cancer risk scores using 165 breast cancer patients and 165 healthy control plasma and used 10 -fold cross-validation to validate the stability of the model performance by age and stage (Table 1).

Plasma samples from breast cancer patients with cancers were obtained from the Korea Regional Biobank of the Korea Institute of Radiological and Medical Sciences, Gangwon National University Hospital, and Inje University Busan Paik Hospital. The breast cancer samples were obtained before any therapeutic approaches were performed. This study was ethically approved by the BIOINFRA Life Science Institutional Review Board (No. 1-700097-B-N-01). The samples were stored at $-80^{\circ} \mathrm{C}$ until analyzed. Plasma samples from asymptomatic healthy donors were obtained from the Korea Regional Biobank of Ajou University Hospital and Kyungpook
National University Hospital. Healthy controls with a known history of cancer, high-grade dysplasia, autoimmune disease, or chronic kidney disease, pregnancy, or inflammatory conditions that needed medical management were excluded. Table 1 presents the number of samples for each age and stage of all breast cancer patients. The cancer clinical stage was determined by the final pathological diagnosis after resection, according to the 7th edition of the Union for International Cancer Control tumor-node-metastasis classification.

\section{Isolation of circulating RNA from plasma}

Circulating RNA was extracted from $300 \mu \mathrm{L}$ of plasma using a nucleic acid automated extraction equipment (Smart Lab Assist-24; Korea KETT, Seoul, Korea), and finally eluted in $150 \mu \mathrm{L}$ of RNase-free water. The concentration and purity of the extracted circulating RNA were confirmed using Thermo Scientific $^{\mathrm{TM}}$ NanoDrop $^{\mathrm{TM}} 2000$ Spectrophotometers (Thermo Fisher Scientific, Waltham, MA, USA).

\section{Removal of genomic DNA (gDNA) and analysis of miRNA gene expression (reverse transcription and quantitative real-time PCR)}

To remove gDNA from the extracted circulating RNA, a gDNA removal process $\left(42^{\circ} \mathrm{C}, 2\right.$ minutes) was performed using gDNA Eraser (product code RR047A, Takara, Shiga, Japan). Quantitative real-time PCR (qRT-PCR) was performed on the total RNA using 4 miRNAs and internal control (IC) primers for standardization. The reaction solution (total RNA, primers, 2X qRT-PCR master mixture, and $\mathrm{ddH}_{2} \mathrm{O}$ ) was put into each of 4 prepared tubes, and qRT-PCR was performed under the following conditions $\left[50^{\circ} \mathrm{C}\right.$ for 15 minutes $(1$ cycle $) \rightarrow 95^{\circ} \mathrm{C}$ for 10 minutes $(1 \mathrm{cycle}) \rightarrow 95^{\circ} \mathrm{C}$ for 10 seconds, and $65^{\circ} \mathrm{C}$ for 20 seconds (40 cycles)] and the Bio-Rad CFX96 Dx system (Bio-Rad, Hercules, CA, USA) was used for genetic analysis. The primer sequences used for PCR follow, and $X$ in the primer sequence represents inosine.

miR-1246 (NR_031648.1) primer sequences: forward, 5'-TCT CTXXXT GAA GTA GGA CTG GGC AGA GA-3'; reverse, 5'-CTC AAXXXT GTT TGC AAT AGC CCT TTG AG-3'

Table 1. Specimen characteristics and breast cancer histology of the subjects used in the generation and validation of models to identify breast cancer risk scores

\begin{tabular}{llcc}
\hline \multicolumn{2}{c}{ Specimens } & $\begin{array}{c}\text { Breast cancers } \\
(\mathrm{n}=165)\end{array}$ & $\begin{array}{c}\text { Healthy controls } \\
(\mathrm{n}=165)\end{array}$ \\
\hline Age & $<50$ & 68 & 60 \\
& 50 to 59 & 50 & 56 \\
Stage & $\geq 60$ & 47 & 49 \\
(average age) & 0 & $5(50.4)$ & - \\
& 1 & $81(52.1)$ & - \\
& 2 & $58(52.6)$ & - \\
& 3 & $21(57.5)$ & - \\
\hline
\end{tabular}

,- not available. 
miR-202 (NR_030170.1) primer sequences: forward, 5'GGC CAXXXG CAT ATA CTT CTT TGA GGA TCT GGC C-3'; reverse, 5'-CAT GGXXXG ACC GCC CCG TTT TCC CAT G-3'

miR-21(NR_029493.1) primer sequences: forward, 5'-CAG TCXXXG TCG GGT AGC TTA TCA GAC TG-3'; reverse, 5'CAG TCXXXC AGA CAG CCC ATC GAC TG-3'

miR-219B (NR 039815.1) primer sequences: forward, 5'ACA TCXXXG GAG CTC AGC CAC AGA TGT-3'; reverse, 5'-GTT TGXXXG CGC CAC TGA TTG TCC AAA C-3'

Human glyceraldehyde-3-phosphate dehydrogenase gene primer sequence: forward, 5'-CAG GTXXXT GCC AAC GTG TCA GTG GTG GAC CTG-3'; reverse, 5'-CAT CCXXXA CCT GGT GCT CAG TGT AGC CCA GGA TG-3'

glyceraldehyde-3-phosphate dehydrogenase was used as the reference gene for qRT-PCR, and RNA concentration used in the experiment was standardized as the reference gene. The Ct (cycle threshold) is defined as the number of cycles required for the fluorescent signal to cross the threshold (ie exceeds background level) and the $\mathrm{dCt}$ is defined as $\mathrm{Ct}$ (reference gene)-Ct (target gene).

\section{Statistical methods}

In this study, the classification performance of 4 miRNA biomarkers was investigated and was particularly high in the patient age group under 50 years old. Before modeling, the differences between breast cancer and healthy controls were examined by age group using box plots and the Mann-Whitney U-test. In addition, we tried 4 classification methods (Iogistic regression, random forest, XGBoost, and generalized linear model plus random forest [GLMRF]) to determine the classification performance of the 4 miRNA biomarkers. These methods are predictive models for screening breast cancer patients and their performance was compared using 10 -fold cross-validation.

Logistic regression is a special case of the general linear model (GLM) and is a representative method used in various classification and prediction models. This method has the advantage of being able to interpret the prediction results through formulas. Random forest and XGBoost are widely used machine learning algorithms as ensemble techniques. In general, these methods are known to have high performance in classification problems, but they have the disadvantages of a high possibility of overfitting and difficulty in interpreting the results. We also used the GLMRF model, which has the advantages of both a linear classification model (explanatory) and a non-linear classification model.

To evaluate and compare the performance of the 4 methods, we used the area under the curve (AUC) and receiver operating characteristic (ROC) curves, which are performance metrics used in classification problems. The ROC curve is a graph that displays the performance for all thresholds at once, and AUC refers to the lower area of the ROC curve. A value closer to 1 indicates an excellent model, and a value closer to 0 indicates a poor model. Detailed performance by age group and the stage was compared using sensitivity, indicating the probability of classifying actual breast cancers as breast cancer, and specificity, indicating the probability of classifying the actual healthy controls as healthy. All analyses were performed using the $\mathrm{R}$ statistical analysis tool (version 4.0.3; R Core Team, Vienna, Austria).
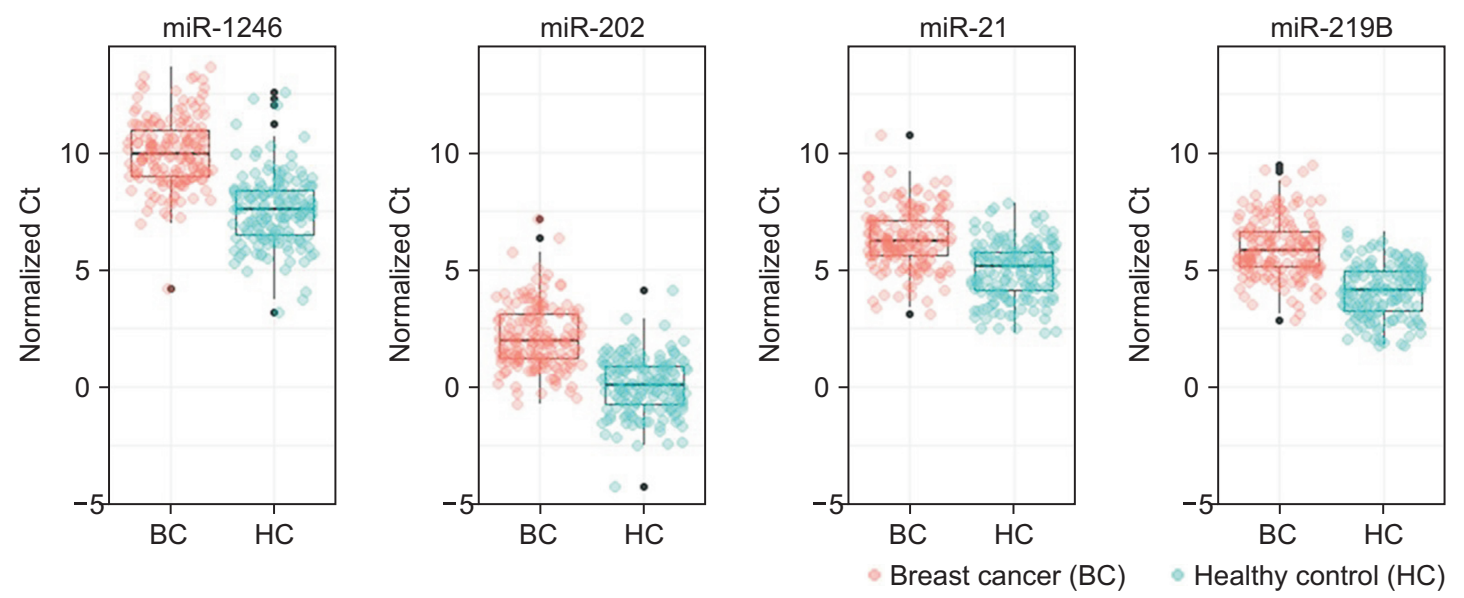

\begin{tabular}{|c|c|c|c|c|}
\hline$P$-value & miR-1246 & miR-202 & miR-21 & miR-219B \\
\hline BC vs. HC & $<2.2 \mathrm{e}-16$ & $<2.2 \mathrm{e}-16$ & $<2.2 \mathrm{e}-16$ & $<2.2 \mathrm{e}-16$ \\
\hline
\end{tabular}

Figure 1. Box plots and Mann-Whitney U-test of single miRNA biomarkers. Each of the 4 miRNAs was indicated to be meaningful in distinguishing healthy controls from patients with breast cancer in box plots. Ct, cycle threshold; miR, microRNA. 


\section{RESULTS}

\section{Performance values for all ages and age} groups for the 4 miRNA biomarkers

We previously reported an optimal panel of multiple biomarkers (miR-1246, miR-206, miR-24 and miR-373) and diagnostic models for screening breast cancer of all ages [17]. In a previous study, it was observed that 4 miRNA biomarkers (miR-1246, 202, 21, 219B) out of a combination of 2 or more miRNA biomarkers out of 9 candidate miRNAs performed well in samples younger than 50 years old (data show that not). In this study, we intended to reaffirm this. In addition, the correlation between miRNAs for these 9 candidate miRNA biomarkers (miR-223, 1246, 206, 24, 373, 21, 6875, 202, 219B) in the previous study was analyzed using Spearman's correlation analysis [17]. It was confirmed that the four miR-
NAs selected for this study had a very low correlation.

We collected breast cancer patients and healthy controls over 20 years of age and performed 4-way modeling to classify breast cancer patients and healthy controls. First, to confirm the performance of each of the 4 miRNA biomarkers, we examined the performance values of each of 4 miRNA biomarkers in 165 breast cancer samples and 165 healthy control samples. The expression level of each miRNA was the intersection of the miRNA amplification curve and the cycle threshold (Ct), which is a relative measure of the target miRNA concentration in the RT-PCR reaction and was internally normalized to control genes. We confirmed that all 4 miRNA biomarkers were reproduced from different samples and were meaningful in differentiating normal and breast cancer patients in box plots (Fig. 1). That is, this result was statistically significant based on the $P$-value.
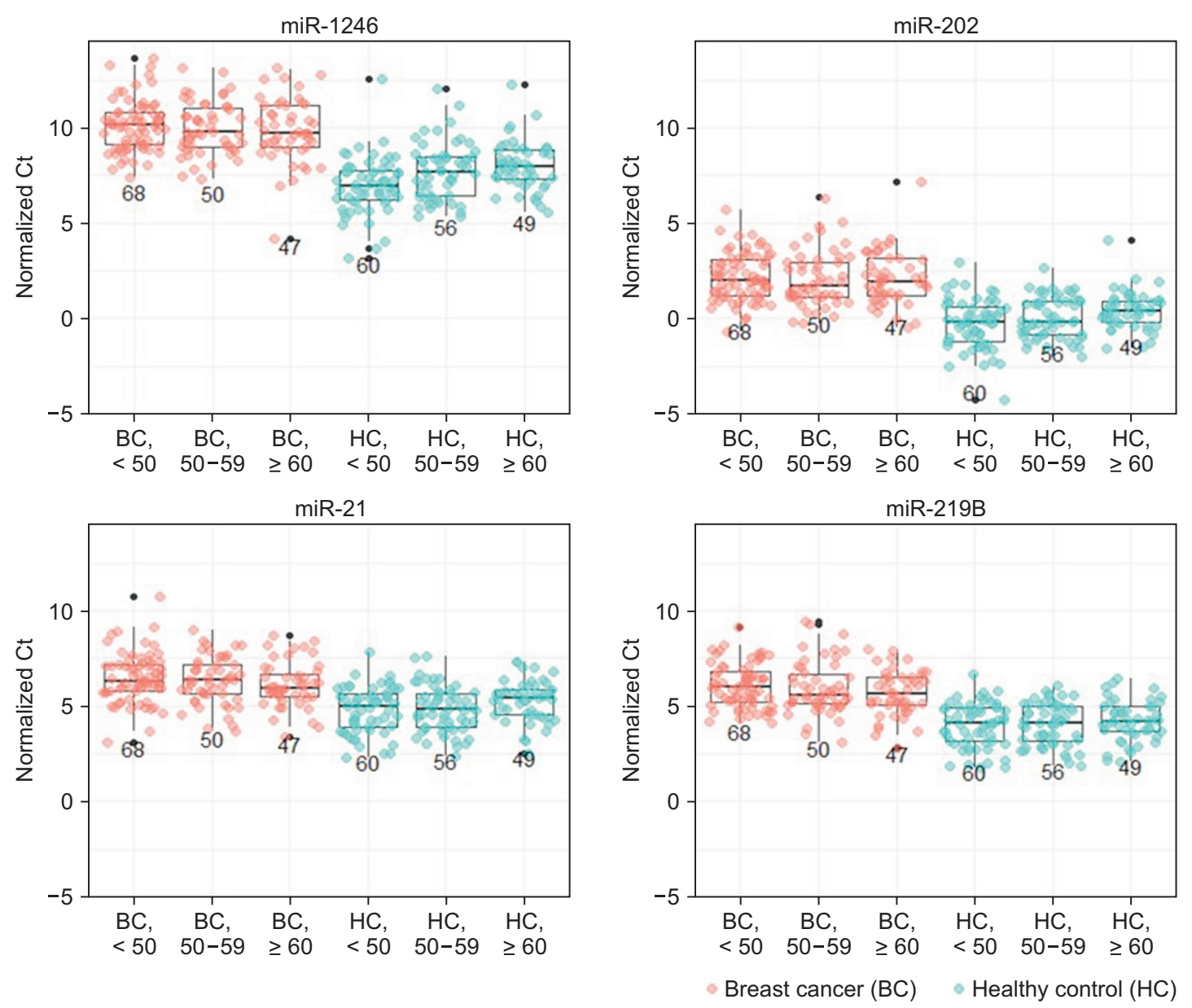

\begin{tabular}{|c|c|c|c|c|}
\hline$P$-value & miR-1246 & miR-202 & miR-21 & miR-219B \\
\hline$<50$ & $<2.2 \mathrm{e}-16$ & $2.58 \mathrm{e}-16$ & $5.887 \mathrm{e}-11$ & $9.03 \mathrm{e}-15$ \\
\hline $50-59$ & $3.75 \mathrm{e}-11$ & $5.157 \mathrm{e}-11$ & $7.728 \mathrm{e}-08$ & $1.012 \mathrm{e}-10$ \\
\hline$\geq 60$ & $1.374 \mathrm{e}-08$ & $1.896 \mathrm{e}-09$ & 0.001052 & $7.16 \mathrm{e}-08$ \\
\hline
\end{tabular}

Figure 2. Box plots and Mann-Whitney U-test of single miRNA biomarkers by age group. Each of the 4 miRNAs was shown to be meaningful in differentiating breast cancer patients from healthy controls in box plot age-specific analysis. Ct, cycle threshold; miR, microRNA. 
The Mann-Whitney U-test for the 4 miRNA biomarkers by age group with box plots confirmed that there was a greater difference between the breast cancer and healthy control groups under the age of 50 (Fig. 2). These results suggest that the 4 miRNA biomarker sets may have higher performance in women under 50 years of age compared to the overall age group. miR-21 is not significant over 60 years of age compared to other miRNA biomakers (Fig. 2). However, in all age groups, inclusion of miR-21 was slightly better than inclusion of miR-21 (Table S1).

\section{Modeling using 4 methods (logistic regression, random forest, XGBoost, and GLMRF) and model validation performance using 10 -fold cross-validation}

To confirm the classification performance of the 4 miRNA biomarker sets in breast cancer and healthy controls under 50 years of age, we performed modeling by applying 4 methods (logistic regression, random forest, XGBoost, and GLMRF). As a model validation method, 10-fold cross-validation was used to improve the stability of the model performance, and AUC and ROC curves were used as performance metrics.
The results confirmed that the performance was excellent in the age group under 50 compared to the overall age in all 4 methods (Fig. 3). Specifically, in logistic regression, the AUC of all age groups was 0.913 and the AUC under 50 was 0.961 , in random forest analysis, the AUC of all ages was 0.903 and the AUC under 50 was 0.967 , and in XGBoost, the AUC of all ages was 0.894 and the AUC under 50 was 0.955 . And for GLMRF, the AUC for all age groups was 0.912 and the AUC under 50 was 0.963 , and AUC was slightly higher for those under 50 than for all age groups in all methods.

\section{Sensitivity and specificity of 4 miRNA biomarkers by the logistic regression model}

To confirm the significance of the 4 miRNA biomarker sets in the early diagnosis of breast cancer under 50 years of age, the sensitivity and specificity by stage and age group were analyzed. The logistic regression results with the highest AUC among the 4 methods tried in this study were examined in detail. The analysis by age group confirmed that the $4 \mathrm{miR}$ NA biomarker sets had a sensitivity of $85.29 \%$ and a specificity of $93.33 \%$ in the age group under 50 years of age, higher than the sensitivity of $82.42 \%$ and specificity of $85.45 \%$ in all
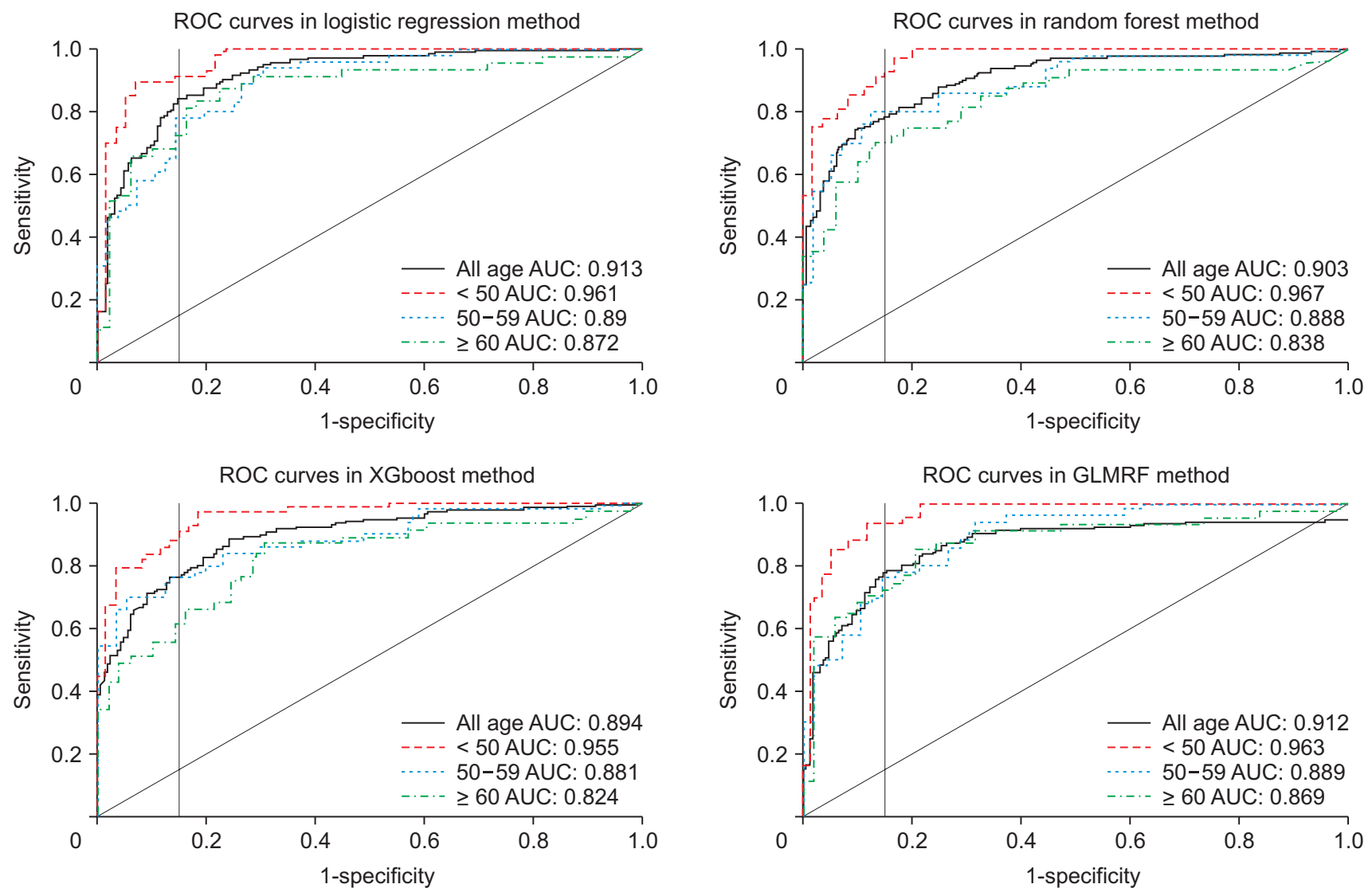

Figure 3. ROC curves of 10 -fold cross-validation for 4 classification methods. Modeling was performed in 4 methods using samples of all ages. The $x$-axis represents the specificity and the $y$-axis represents the sensitivity. The combination of 4 miRNA biomarkers had the highest performance in patients under 50 years of age. ROC curve, receiver operating characteristic curve; AUC, area under the curve; GLMRF, generalized linear model plus random forest. 
Table 2. Sensitivity and specificity by age and stage in the logistic regression method

\begin{tabular}{llcc}
\hline \multirow{2}{*}{ Specimens } & \multicolumn{2}{c}{ Logistic regression method $(\mathrm{n}=165)$} \\
\cline { 3 - 4 } & & Specificity $(\%)$ & Sensitivity $(\%)$ \\
\hline \multirow{2}{*}{ Total } & & 85.45 & 82.42 \\
Age & $<50$ & 93.33 & 85.29 \\
& 50 to 59 & 80.36 & 78.00 \\
& $\geq 60$ & 81.63 & 82.98 \\
Stage & 0 & - & 60.00 \\
& 1 & - & 86.42 \\
& 2 & - & 79.31 \\
& 3 & - & 80.95 \\
\hline
\end{tabular}

-, not available.

ages (Table 2). These results are very meaningful, showing the possibility that a set of 4 miRNA biomarkers could provide supplemental data for the low sensitivity of mammography in Korean women under 50 years of age. And, analysis by age group included stages $0,1,2$, and 3 samples, the early stages of breast cancer correspond to stages 0,1 , and 2 , and the number of stage 0 samples used in this study is too small to determine the accuracy of stage 0 . In the case of stage 3 , the number of samples was smaller than that of stage 1 and stage 2, and the average patient age was higher than that in stage 1 and stage 2, indicating that the sensitivity was affected. In the 4 miRNA biomarkers, the sensitivity was $86.42 \%$ in stage 1 and $79.31 \%$ in stage 2, confirming that it was higher or similar to the sensitivity of $82.42 \%$ for all stages (Table 2). That is, the four miRNA biomarker sets showed the potential to compensate for the low sensitivity of the existing breast cancer screening method with an average sensitivity of $83.45 \%$ for stage 1 and stage 2 breast cancer. That is, the set of 4 miRNA biomarkers showed the potential to compensate for the low sensitivity of mammography with an average sensitivity of $83.45 \%$ for stages 1 and 2 breast cancer.

\section{DISCUSSION}

The early diagnosis of breast cancer is difficult because there are no symptoms in the early stages. Patients with early-detected breast cancer have better treatment outcomes and higher survival rates than other cancers, but the survival rate is low in patients with terminal cancer, so accurate early diagnosis is very important.

Mammography is the only screening test scientifically proven to reduce breast cancer mortality. However, mammography has disadvantages such as hospitalization, psychological burden, and radiation exposure, as it can lead to false-negative and false-positive diagnoses, which can lead to excessive additional tests and unnecessary biopsies [18]. Above all, the sensitivity of mammography is inversely proportional to breast density. Korean women aged 40 to 49 years, who account for more than $70 \%$ of high-density breasts, have low- er sensitivity in mammography than women aged 50 years or older. Therefore, breast cancer screening by mammography was associated with a significant decrease in mortality in patients aged 50 years and older, but this difference was not statistically significant when only women aged 40 to 49 years were included. Even under 40 years of age, breast cancer screening by mammography did not reduce breast cancer mortality $[19,20]$ according to the results of a comparative evaluation of the accuracy of screen-film mammography (SFM) and full-field digital mammography (FFDM) for breast cancer screening in more than 8 million Korean women. Sensitivity and positive predictive value (PPV) were higher in FFDM than in SFM, but specificity was lower in FFDM, and the overall AUC of FFDM was 0.80, higher than that of SFM at 0.75 . In particular, in the case of patients under 50 years of age, the sensitivity of SFM was $49.0 \%$ to $53.7 \%$ and the specificity was $86.0 \%$ to $86.2 \%$. In the case of FFDM, the sensitivity in women under the age of 50 was $54.6 \%$ to $54.5 \%$ and the specificity was $85.3 \%$ to $86.0 \%$, which was better than SFM, but the sensitivity was still low [21]. These results show the importance of developing a new auxiliary diagnostic method that can improve the sensitivity of mammography and are considered important reference material for evaluating the significance of the results of this study.

Breast ultrasonography is mainly used as an auxiliary test to compensate for the problems of mammography. When both mammography and ultrasonography are performed as screening tests, the sensitivity is improved compared to when only mammography is performed. However, despite the development of breast ultrasound equipment, this auxiliary test also has limitations due to the high dependence of the diagnosis on the examiner and difficulty in the early diagnosis of breast cancer due to calcified lesions [22].

As a new screening method for high-risk breast cancer that can assist with mammography, liquid biopsy is non-invasive and minimizes the inconvenience of an examination [23]. In this study, we tried to verify the combination of multiple miRNA biomarkers with high sensitivity in women under the age of 50 with a high percentage of dense breasts, which are difficult to accurately diagnose by mammography.

miRNAs used as breast cancer screening biomarkers have demonstrated the value of circulating miRNAs in breast cancer diagnosis in several previous studies [24,25]. However, there have been no reports on miRNAs that significantly selected Korean breast cancer patients under 50 years of age. In this study, the expression levels of 4 miRNAs in plasma were analyzed to verify whether they significantly identified Korean breast cancer patients under 50 years of age. In particular, in this study, we used a dumbbell-shaped rescue primer for pre-miRNA amplification by RT-PCR, our team's proprietary technology that can minimize non-specific PCR in real time. Previous studies reported that the 4 miRNAs used in this study were involved in breast cancer development and progression. However, in contrast to our study, the published 
Jang et al.

papers analyzed mature miRNA expression [26-30].

The results of this study showed that the AUC value of the 4 miRNA biomarkers (miR-1246, miR-202, miR-21, and miR-219B) measured in plasma for the early diagnosis of breast cancer was 0.913 in the logistic method. In particular, under the age of 50 , the AUC was 0.961 , the sensitivity was $85.29 \%$, and the specificity was $93.33 \%$, showing the highest performance.

It is not yet known whether the developed multi-miRNA set can discriminate between breast cancer and benign breast disease. In particular, the selectability of breast cancer and difficult-to-selectable mammary glands and benign diseases in mammography should be studied using samples from patients with benign breast calcifications in the future.

In conclusion, the 4 miRNA biomarker set was found to be meaningful in the early diagnosis of breast cancer in Korean women under 50 years of age. The set of 4 miRNA biomarkers provided higher sensitivity and specificity in the age group of patients under 50 years compared to all age groups, suggesting that it may be helpful in supplementing mammography sensitivity in Korean women under the age of 50 with a high percentage of dense breasts. For reference, miR-21 among the 4 miRNA biomarkers showed significant performance in other carcinomas, whereas the other three miRNAs did not (data not shown).

The clinical significance of the results of this study is that it can provide a basis for the development of a new adjunct tool to improve the accuracy of mammography and hence can be utilized as a new high-accuracy breast cancer screening tool. Therefore, it is expected that the treatment effect and survival rate of breast cancer can be improved. However, many factors must be considered for clinically use, and additional validation of an appropriate screening population through largescale clinical trials is required.

\section{CONFLICTS OF INTEREST}

No potential conflicts of interest were disclosed.

\section{SUPPLEMENTARY MATERIALS}

Supplementary materials can be found via https://doi. org/10.15430/JCP.2021.26.4.258.

\section{ORCID}

Ji Young Jang, https://orcid.org/0000-0003-4347-9245

Eun Young Ko, https://orcid.org/0000-0001-9406-4395

Ji Soo Jung, https://orcid.org/0000-0001-7230-9099

Kyung Nam Kang, https://orcid.org/0000-0002-2976-3920

Yeon Soo Kim, https://orcid.org/0000-0002-6516-7085

Chul Woo Kim, https://orcid.org/0000-0002-1229-198X

\section{REFERENCES}

1. Hong S, Won YJ, Park YR, Jung KW, Kong HJ, Lee ES. Cancer Statistics in Korea: incidence, mortality, survival, and prevalence in 2017. Cancer Res Treat 2020;52:335-50.

2. Jung KW, Won YJ, Kong HJ, Lee ES. Prediction of cancer incidence and mortality in Korea, 2019. Cancer Res Treat 2019;51:431-7.

3. Lee JE, Lee SA, Kim TH, Park S, Choy YS, Ju YJ, et al. Projection of breast cancer burden due to reproductive/lifestyle changes in Korean women (2013-2030) using an age-periodcohort model. Cancer Res Treat 2018;50:1388-95.

4. Yoo KY. Cancer control activities in the Republic of Korea. Jpn J Clin Oncol 2008;38:327-33.

5. Lee EH, Park B, Kim NS, Seo HJ, Ko KL, Min JW, et al. The Korean guideline for breast cancer screening. J Korean Med Assoc 2015;58:408-19.

6. Ciatto S, Visioli C, Paci E, Zappa M. Breast density as a determinant of interval cancer at mammographic screening. $\mathrm{Br} \mathrm{J}$ Cancer 2004;90:393-6.

7. Youk JH, Kim EK. Supplementary screening sonography in mammographically dense breast: pros and cons. Korean J Radiol 2010;11:589-93.

8. Winkler NS, Raza S, Mackesy M, Birdwell RL. Breast density: clinical implications and assessment methods. Radiographics 2015;35:316-24.

9. Harvey JA, Bovbjerg VE. Quantitative assessment of mammographic breast density: relationship with breast cancer risk. Radiology 2004;230:29-41.

10. McCormack VA, Perry N, Vinnicombe SJ, Silva Idos S. Ethnic variations in mammographic density: a British multiethnic longitudinal study. Am J Epidemiol 2008;168:412-21.

11. Ellison-Loschmann L, McKenzie F, Highnam R, Cave A, Walker $\mathrm{J}$, Jeffreys M. Age and ethnic differences in volumetric breast density in new zealand women: a cross-sectional study. PLoS One 2013;8:e70217.

12. Youn I, Choi S, Kook SH, Choi YJ. Mammographic breast density evaluation in Korean women using fully automated volumetric assessment. J Korean Med Sci 2016;31:457-62.

13. Jeon JH, Kang JH, Kim Y, Lee HY, Choi KS, Jun JK, et al. Reproductive and hormonal factors associated with fatty or dense breast patterns among Korean women. Cancer Res Treat 2011;43:42-8.

14. Byrne C. Studying mammographic density: implications for understanding breast cancer. J Natl Cancer Inst 1997;89:531-3.

15. Boyd NF, Lockwood GA, Byng JW, Tritchler DL, Yaffe MJ. Mammographic densities and breast cancer risk. Cancer Epidemiol Biomarkers Prev 1998;7:1133-44.

16. Lam PB, Vacek PM, Geller BM, Muss HB. The association of increased weight, body mass index, and tissue density with the risk of breast carcinoma in Vermont. Cancer 2000;89:369-75.

17. Jang JY, Kim YS, Kang KN, Kim KH, Park YJ, Kim CW. Multiple microRNAs as biomarkers for early breast cancer diagnosis. Mol Clin Oncol 2021;14:31. 
18. Løberg M, Lousdal ML, Bretthauer M, Kalager M. Benefits and harms of mammography screening. Breast Cancer Res 2015;17:63.

19. Kim SH, Kim MH, Oh KK. Analysis and comparison of breast density according to age on mammogram between Korean and Western women. J Korean Radiol Soc 2000;42:1009-14.

20. Kim EH, Bae JM. Potential availability of dense mammogrphy for prevention of breast cancer in Korean women. Korean J Fam Pract 2014;4:181-5.

21. Hong S, Song SY, Park B, Suh M, Choi KS, Jung SE, et al. Effect of digital mammography for breast cancer screening: a comparative study of more than 8 million Korean women. Radiology 2020;294:247-55.

22. Guo R, Lu G, Qin B, Fei B. Ultrasound imaging technologies for breast cancer detection and management: a review. Ultrasound Med Biol 2018;44:37-70.

23. Alimirzaie $S$, Bagherzadeh M, Akbari MR. Liquid biopsy in breast cancer: a comprehensive review. Clin Genet 2019;95:643-60.

24. Hamam R, Hamam D, Alsaleh KA, Kassem M, Zaher W, Alfayez $M$, et al. Circulating microRNAs in breast cancer: novel diagnostic and prognostic biomarkers. Cell Death Dis 2017;8:e3045.

25. He Y, Deng F, Yang S, Wang D, Chen X, Zhong S, et al. Exosomal microRNA: a novel biomarker for breast cancer. Biomark Med 2018;12:177-88.

26. Fu L, Li Z, Zhu J, Wang P, Fan G, Dai Y, et al. Serum expression levels of microRNA-382-3p, -598-3p, -1246 and -184 in breast cancer patients. Oncol Lett 2016;12:269-74.

27. Hannafon BN, Trigoso YD, Calloway CL, Zhao YD, Lum DH, Welm AL, et al. Plasma exosome microRNAs are indicative of breast cancer. Breast Cancer Res 2016;18:90.

28. Asaga S, Kuo C, Nguyen T, Terpenning M, Giuliano AE, Hoon DS. Direct serum assay for microRNA-21 concentrations in early and advanced breast cancer. Clin Chem 2011;57:84-91.

29. Schrauder MG, Strick R, Schulz-Wendtland R, Strissel PL, Kahmann L, Loehberg CR, et al. Circulating micro-RNAs as potential blood-based markers for early stage breast cancer detection. PLoS One 2012;7:e29770.

30. Kim J, Park S, Hwang D, Kim SI, Lee H. Diagnostic value of circulating miR-202 in early-stage breast cancer in South Korea. Medicina (Kaunas) 2020;56:340. 